\title{
Structure and stability of Mg-intercalated boron nanotubes and crystalline bundles
}

\author{
Kah Chun Lau, Roberto Orlando ${ }^{1}$ and Ravindra Pandey ${ }^{2}$ \\ Department of Physics, Michigan Technological University, Houghton, MI 49931, USA \\ E-mail: pandey@mtu.edu
}

Received 11 September 2008, in final form 16 November 2008

Published 22 December 2008

Online at stacks.iop.org/JPhysCM/21/045304

\begin{abstract}
First principles calculations based on density functional theory predict a highly selective adsorption site for $\mathrm{Mg}$ atoms and negligible preference for the growth of $\mathrm{Mg}$ islands on the tubular surface of Mg-intercalated (small diameter) boron nanotubes, thereby establishing the criterion for understanding the growth mechanism of single-walled boron nanotubes (SWBNTs) supported by magnesium. On the other hand, the Mg-SWBNT bundles can be considered as an 'electrostatic' bound system consisting of partially ionized $\mathrm{Mg}$ and partially ionized tubules. The metallic character of the tubular $\mathrm{Mg}-\mathrm{B}$ bundles is then attributed to boron atoms forming a metallic wire, while the role of $\mathrm{Mg}$ atoms is limited in enhancing the stability of the crystalline bundles.
\end{abstract}

(Some figures in this article are in colour only in the electronic version)

\section{Introduction}

In recent years nanostructures based on elemental boron have been the focus of several theoretical studies which have predicted novel properties associated with such nanostructures [1-10]. For example, single-walled boron nanotubes (SWBNTs) were found to be associated with a very low Poisson ratio [4] and ballistic conduction for electron transport [6]. It was also indicated that boron nanostructures are different [4, 5, 7-9] from $\mathrm{sp}^{2}$-bonded planar structures, such as carbon and boron nitride. The instability of a graphene-like boron sheet is attributed to the multi-centered bonds and electron-deficient features of boron which are energetically more competitive and stable than the $\mathrm{sp}^{2}$ bonding features. In crystalline bundles of boron nanotubes, the intertubular interaction is predicted to be rather strong and peculiar $[7,11,12]$, and the competing interplay among intratubular and intertubular interactions seems to be responsible for the predicted polymorphism [12]. The experimental way to synthesize elemental boron nanotubes can, therefore, be rather challenging.

Crystalline bundles of SWBNTs offer an all-boron host lattice for intercalation and energy storage. Following the

\footnotetext{
1 Permanent address: Dipartimento di Scienze e Tecnologie Avanzate, Università del Piemonte Orientale, Via Bellini 25/G, 15100 Alessandria, Italy.

2 Author to whom any correspondence should be addressed.
}

analogy of the Li-intercalated nanorope crystallites of carbon nanotubes [13], boron nanotube bundles comprising uniform 1D metallic SWBNTs [12] are expected to be ideal candidates for anode materials in nanoscopic ion battery applications. In this context, the precise determination of the intercalation sites of metal cations in SWBNT crystalline bundles is essential for the design of such electrodes. First principles studies can now routinely perform the task of predicting energetically competitive atomic configurations, and related electronic properties in nanostructures. In this study we propose to do such calculations for $\mathrm{Mg}$-intercalated crystalline tubular bundles of elemental boron. Specifically, we will study the interaction between a $\mathrm{Mg}$ atom and SWBNTs to determine the energetically preferred intercalation site in SWBNTs and bundles. Note that synthesis of SWBNTs using a mesoporous silica (MCM-41) template with magnesium as the catalyst or precursor was recently reported [14].

\section{Method}

First principles calculations were performed in the framework of the all-electron density functional theory (DFT) with the Perdew-Wang 91 exchange-correlation functional form [15]. The periodic linear combination of atomic orbitals (LCAO) approximation as embedded in the CRYSTAL06 [16] program was employed. A linear combination of Gaussian-type orbitals 

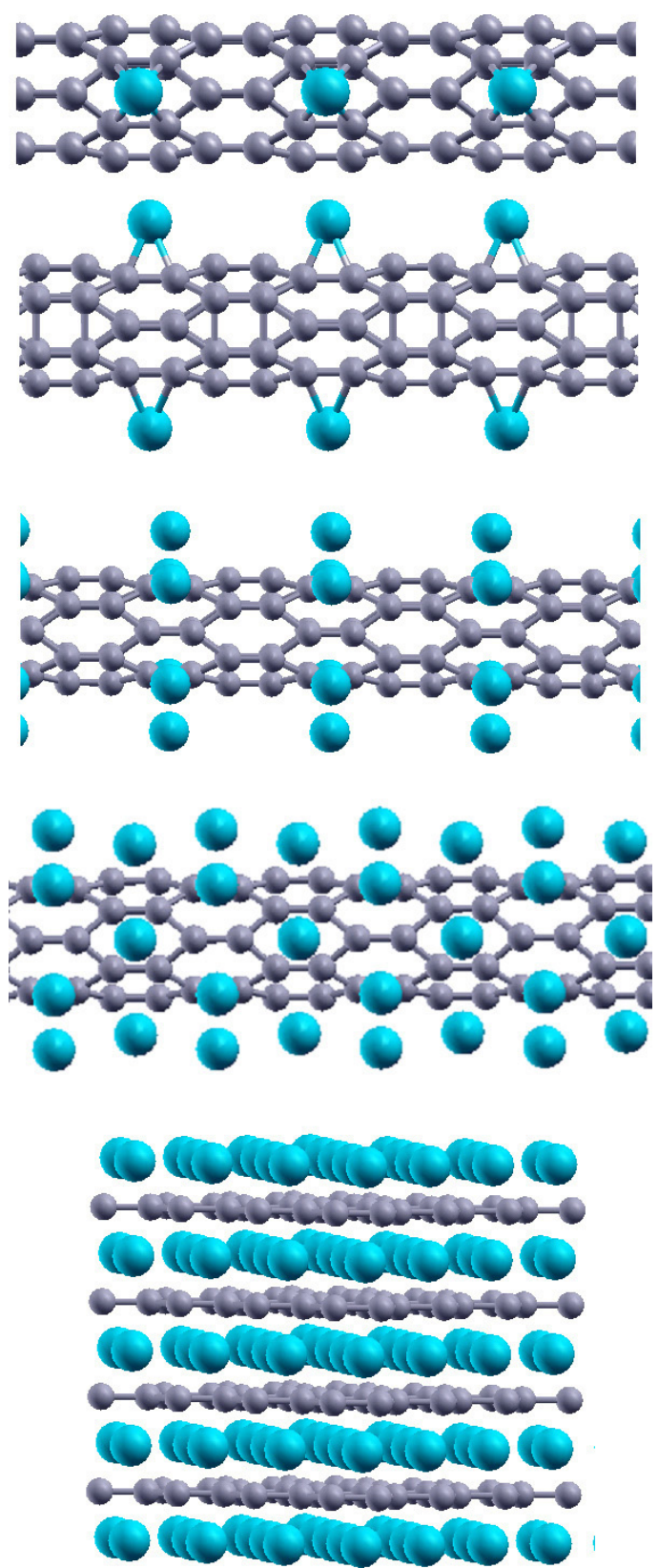

Figure 1. The $\mathrm{MgB}_{2}$ solid (bottom) and single-walled boron nanotubes with $\mathrm{Mg}$ (from top to bottom): $\Theta$, coverage (table 1) is (1:24), (1:12), (1:6), (1:4) and (1:2).

(GTOs) was used to construct a localized atomic basis from which Bloch functions were constructed by a further linear combination with the plane-wave phase factors.

A split-valence basis set with polarization functions (i.e. 6-31G(d, p)) was used [12]. With periodicity along the $x$-axis for pristine SWBNT, the $1 \times 8 \times 8$ MonkhorstPack grid is employed. While for the $3 \mathrm{D}$ crystalline SWBNT bundles, the Brillouin zone was sampled using an $8 \times 8 \times 8$ Monkhorst-Pack grid for integration in the reciprocal space. A total energy tolerance of $10^{-7}$ Hartree, and eigenvalue tolerance of $10^{-6}$ Hartree in the iterative solution of the KohnSham equations were set. The reliability and accuracy of the computational model employed were successfully tested on the well-studied boron crystalline solid $\alpha-\mathrm{B}_{12}$ and bulk magnesium, whose structural and electronic properties were reproduced by the modeling elements employed [12, 17].

The lattice parameters as well as the internal coordinates at each fixed value of the crystallographic unit-cell volume were optimized for the tubular configuration. The tubes are of infinite length and not capped. The crystalline bundles of SWBNTs were represented by arrays of identical nanotubes arranged in a hexagonal lattice. In a hexagonal unit cell, $a$ is defined as the sum of the diameter of the nanotube and its intertubular distance, and $c$ represents the periodicity of a SWBNT along its tubular axis. The calculated results for the unit cell chosen were compared with those obtained using a supercell three-times larger $\left(a^{\prime}=a, c^{\prime}=3 c\right)$. The difference between these two sets of calculations for $\mathrm{Mg}$ intercalated SWBNTs was found to be very small suggesting a negligible influence of the adsorbate-adsorbate interaction in the calculated results presented.

\section{Results and discussion}

\subsection{Mg-doped pristine SWBNTs}

We begin with calculations to find the binding sites for a magnesium atom on the tubular surface. The chosen tubular configuration was a $(6,0)$ zigzag type-I SWBNT with a diameter of about $4 \AA$. This is one of the most stable configurations predicted by first principles calculations [5, 12], derived from the reconstructed $\{1221\}$ boron sheet $^{3}[5,9,18]$. The SWBNT is characterized in a hexagonal lattice with the space group $P 6 / \mathrm{mmm}$. On the tubular surface, the binding sites can be classified as $\mathrm{H}$ (above the center of the hexagons), A (above the axial B-B bonds), $\mathrm{Z}$ (above the zigzag triangles) and $\mathrm{T}$ (above a boron atom) sites. We define the binding energy $\left(E_{\mathrm{b}}\right)$ of a $\mathrm{Mg}$ atom adsorbed at a given site to be the difference in energy of the combined system and that of individual constituents. A positive value of the binding energy suggests that $\mathrm{Mg}$ is bound at a given site on the tubular surface.

Analogous to the bulk crystalline $\mathrm{MgB}_{2}$ solid, which favors the $\mathrm{Mg}$ atom sitting over the center of hexagonal sites [20], the $\mathrm{H}$ site on the tubular surface (figure 1) is predicted to be the energetically most favorable site for the adsorption of $\mathrm{Mg}$ with $E_{\mathrm{b}}$ of $\sim 1.55 \mathrm{eV}$. On the other hand, $\mathrm{Mg}$ does not seem to prefer adsorption at $\mathrm{T}, \mathrm{A}$ and $\mathrm{Z}$ sites, and is associated with the negative values of $E_{\mathrm{b}}$ (i.e. $\sim-0.42 \mathrm{eV}$ ) at $\mathrm{T}$ sites, and weakly bound at $\mathrm{A}$ and $\mathrm{Z}$ sites (i.e. $E_{\mathrm{b}} \sim$ 0.21 and $0.20 \mathrm{eV}$, respectively). This is in contrast to the case of Al-doped $(8,0)$ zigzag single-walled carbon nanotubes (SWCNTs) where the $\mathrm{H}, \mathrm{Z}$ and A sites are the stable sites for $\mathrm{Al}$ adsorption on the tubular surface [21]. The adsorption of $\mathrm{Mg}$ on a SWBNT is therefore much more selective than that of $\mathrm{Al}$ on a SWCNT of a similar chirality and diameter. We note that encapsulation of a $\mathrm{Mg}$ atom inside the nanotube was also considered. It was found to be energetically unfavorable with $E_{\mathrm{b}} \sim-0.1 \mathrm{eV}$. We believe that the instability is likely to

3 Calculations find a significant structural deformation on the surface of (6, 0) zigzag type-III SWBNTs upon Mg adsorption. Type-III SWBNTs were derived from a triangular lattice refereed to as the $\{1212\}$ boron sheet in our previous work $[5,9,12]$. 

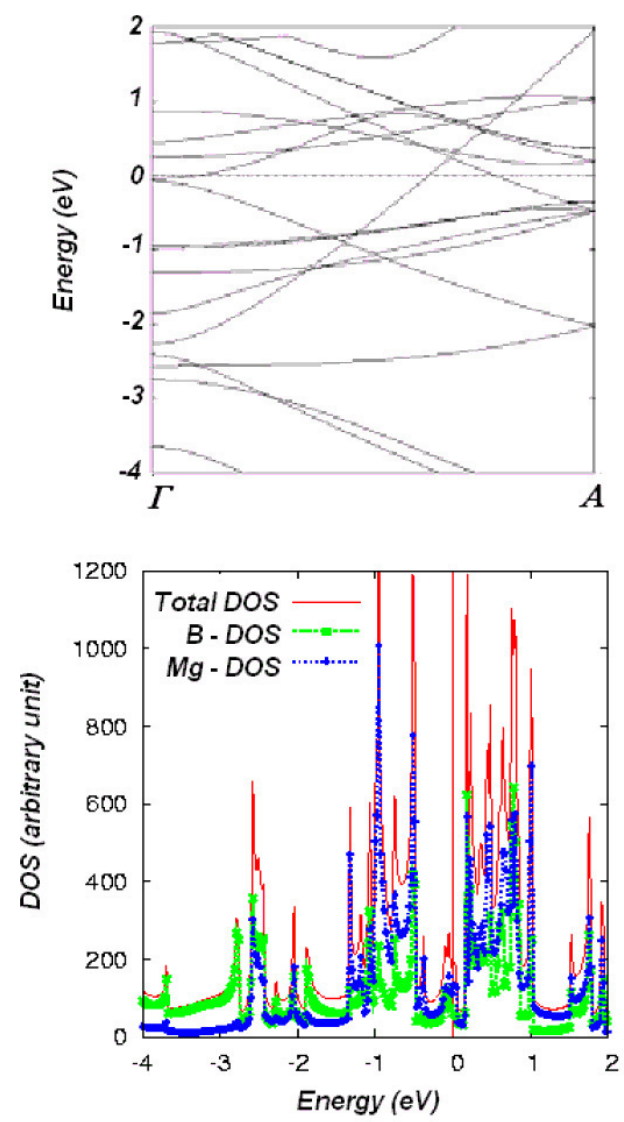

(2a)
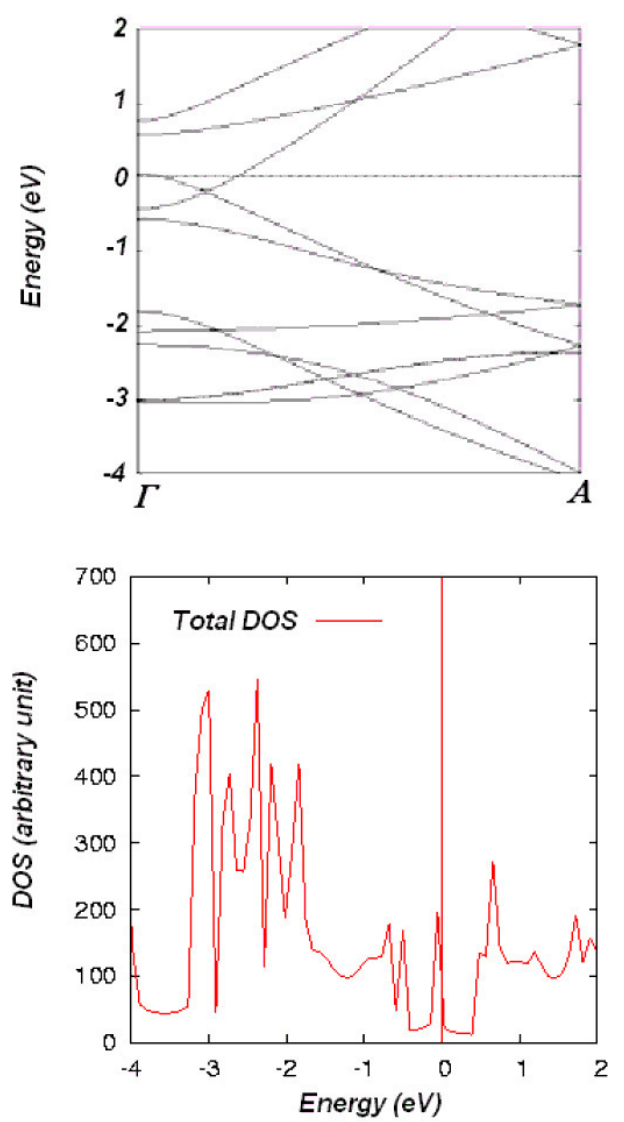

(2b)

Figure 2. Band structures and density of states of Mg-SWBNT with $\Theta=(1: 2)$ (left), and pristine SWBNTs (right). The Fermi level is shifted to $0 \mathrm{eV}$.

be due to a steric repulsion arising from a smaller diameter of about $4 \AA$ of the SWBNT considered.

Considering only the $\mathrm{H}$ site to be the binding site for $\mathrm{Mg}$, we now increase the coverage of $\mathrm{Mg}$ atoms to study the interplay between $\mathrm{Mg}-\mathrm{Mg}, \mathrm{B}-\mathrm{B}$ and $\mathrm{Mg}-\mathrm{B}$ interaction in determining the stability of tubular configurations. For the coverage of $\Theta=\frac{1}{12}$, where $\Theta$ is the ratio of $\mathrm{B}: \mathrm{Mg}$ in the unit cell, the optimized configuration shows both $\mathrm{Mg}$ atoms to be bonded at the opposite $\mathrm{H}$ sites (figure 1). As we increase the coverage to $\Theta=\frac{1}{4}$ by placing the six $\mathrm{Mg}$ atoms at the $\mathrm{H}$ sites around the circumference of the tube (figure 1), the equilibrium configuration does not show any preference for forming $\mathrm{Mg}$ dimers at the surface. For the maximum uniform coverage of $\Theta=\frac{1}{2}$ the equilibrium configuration exhibits its stability without forming islands of $\mathrm{Mg}$ atoms (figure 1). The results suggest that the $\mathrm{Mg}-\mathrm{B}$ interactions appear to be dominant relative to the $\mathrm{Mg}-\mathrm{Mg}$ interaction in these tubular nanostructures ${ }^{4}$. The relatively high stability of $\mathrm{Mg}$ atoms on the tubular surface without deforming the SWBNT configuration together with negligible preference for growth

\footnotetext{
4 The strength of the interaction can be directly related to the bond distances. In the $\mathrm{MgB}_{2}$ solid, $\mathrm{Mg}-\mathrm{B}$ and $\mathrm{Mg}-\mathrm{Mg}$ bond lengths are 2.49 and $3.05 \AA$, respectively compared to the experimental values [19] of 2.51 and $3.09 \AA$. For the $\mathrm{Mg}-\mathrm{SWBNT}$ configuration with $\Theta=\frac{1}{2}$, the $\mathrm{Mg}-\mathrm{B}$ and $\mathrm{Mg}-\mathrm{Mg}$ bond distances are 2.53 and $3.6 \AA$, respectively.
}

of $\mathrm{Mg}$ islands can be comprehended as an important criterion for modeling the SWBNT growth mechanism supported by magnesium as the catalyst or precursor.

The band structure and density of states near the Fermi level $\left(E_{\mathrm{F}}\right)$ of pristine and $\mathrm{Mg}-\mathrm{SWBNT}$ configurations are given in figure 2. For pristine SWBNTs, the top of the valence band and bottom of the conduction band are highly dispersive with significant delocalized p-character, though the inner valence bands associated with the localized $\sigma$-bonds are rather narrow and flat. The SWBNTs considered are predicted to be metallic and possess $2 G_{0}$ (i.e. $G_{0}=\frac{2 e^{2}}{\hbar}$ ) in their intrinsic conductance $[5,6,12]$. As the SWBNTs are uniformly covered by $\mathrm{Mg}$ atoms, significant changes occur in the electronic structure of Mg-SWBNT configurations. For example, the $\pi$ bonds around the tube circumference due to the $\mathrm{B}$ p-character together with $\mathrm{Mg}$ atoms surrounding the tube yield rich features in the band structure and DOS for the case of $\Theta=\frac{1}{2}$ in the vicinity of $E_{\mathrm{F}}$ (figure 2). The upward shift of $E_{\mathrm{F}}$ in $\mathrm{Mg}-\mathrm{SWBNT}$ relative to pristine SWBNTs causes partial occupancy of a number of highly dispersive bands crossing $E_{\mathrm{F}}$ resulting into a considerably higher conductivity. Multiple bands cross at $E_{\mathrm{F}}$, thereby allowing electron transport in several modes in these tubular configurations.

Mulliken charge analysis indicates a charge transfer of $\sim 0.20$ e from each $\mathrm{Mg}$ atom to the $\mathrm{B}$ sublattice. This is 

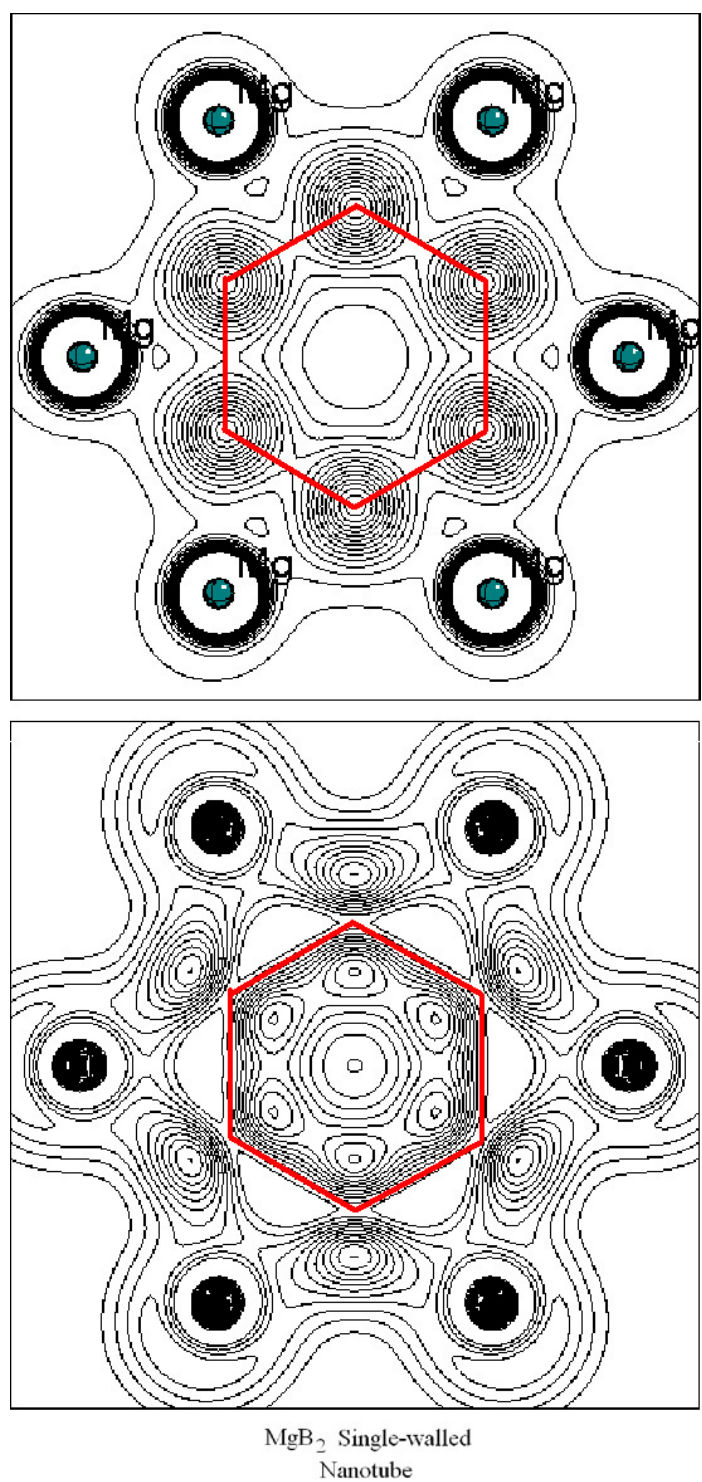

Figure 3. The 2D contour map of Mg-SWNT with $\Theta=(1: 2)$. Top: total charge density (iso-density curves taken at levels differing from each other by $10^{-2} \mathrm{au}$ ). Bottom: the partially occupied conduction level showing the overlap of B p-character and $\mathrm{Mg}$ s-character (iso-density curves at levels differing by $5 \times 10^{-5} \mathrm{au}$ ).

consistent with the prediction of the rigid shift of the Fermi level of $\mathrm{Mg}-\mathrm{SWBNT}$ configurations via electron injection from $\mathrm{Mg}$ atoms ${ }^{5}$. A cross-sectional view of the tubular configuration with $\Theta=\frac{1}{2}$ in a $2 \mathrm{D}$ contour plot is shown in figure 3 . The contours show a spherically symmetric localized region around $\mathrm{Mg}$, indicating the bonding between $\mathrm{Mg}$ and $\mathrm{B}$ to be partially ionic.

\subsection{Mg-intercalated crystalline bundles of SWBNTs}

In the crystalline bundles of small diameter SWBNTs considered, the preferred site for the intercalation of $\mathrm{Mg}$ atoms

5 The indirect evidence of electron injection from $\mathrm{Mg}$ atoms onto SWBNTs can also be comprehended as the shift in Fermi level $E_{\mathrm{F}}$ of a SWBNT (i.e. $E_{\mathrm{F}}^{(\mathrm{SWBNT})} \sim-0.162$ Hartree) towards the unoccupied conduction bands following the increase in the $\mathrm{Mg}$ ratio, $\Theta$ (i.e. $E_{\mathrm{F}}^{(\Theta=1 / 2)} \sim-0.108$ Hartree).
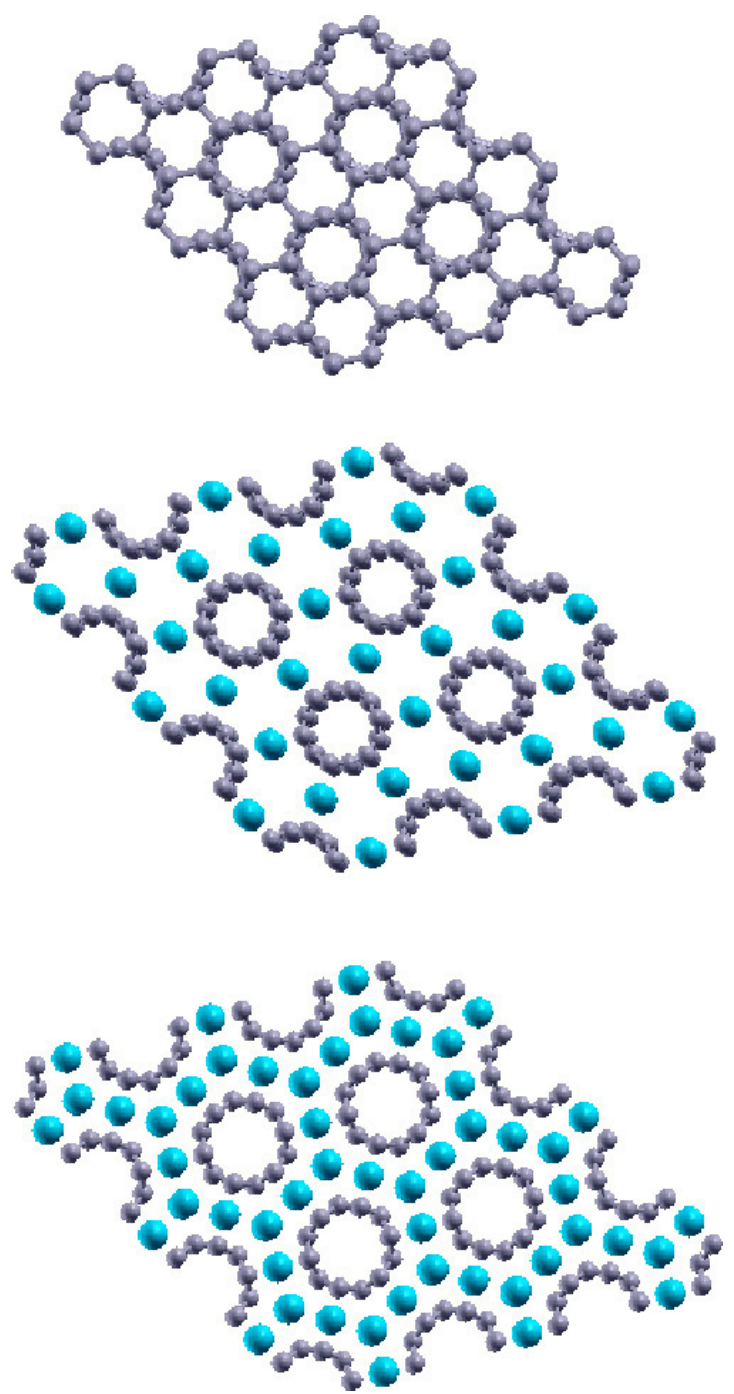

Figure 4. The equilibrium configurations of (top) SWBNT bundles (center) $\mathrm{Mg}$-intercalated crystalline bundles- $\mathrm{MgB}_{8}$, and (bottom) $\mathrm{Mg}_{5} \mathrm{~B}_{24}$ bundles.

is the high symmetry interstitial site as shown in figure 4. Table 2 collects the calculated results for the intercalation coverage, $\Theta(\mathrm{Mg}: \mathrm{B})=1: 8$ and 5:24, respectively. We note the choice of these coverages reflects the limit of our computational resources. Nonetheless, the results would shed light on the variation of the properties with the increasing intercalation density of $\mathrm{Mg}$ atoms in SWBNT bundles.

In the pristine crystalline bundles, the intertubular covalent bonds are predicted to be dominant [12]. As $\mathrm{Mg}$ atoms are intercalated at the interstitial sites in the lattice, the intertubular distance increases, resulting into weakening of two-center and three-center bonds among the neighboring tubules [12]. There is, however, a small contraction in the lattice volume (table 2) when we increase $\Theta$ from $(1: 8)$ to $(5: 24)$. This is due to the fact that the electrostatic interaction between the partially ionized $\mathrm{Mg}$ and the negatively charged tubules increases with the increase in the intercalation density, which in turn contracts the lattice. These competing factors yield a nearly constant intercalation energy for the coverage cases considered. 

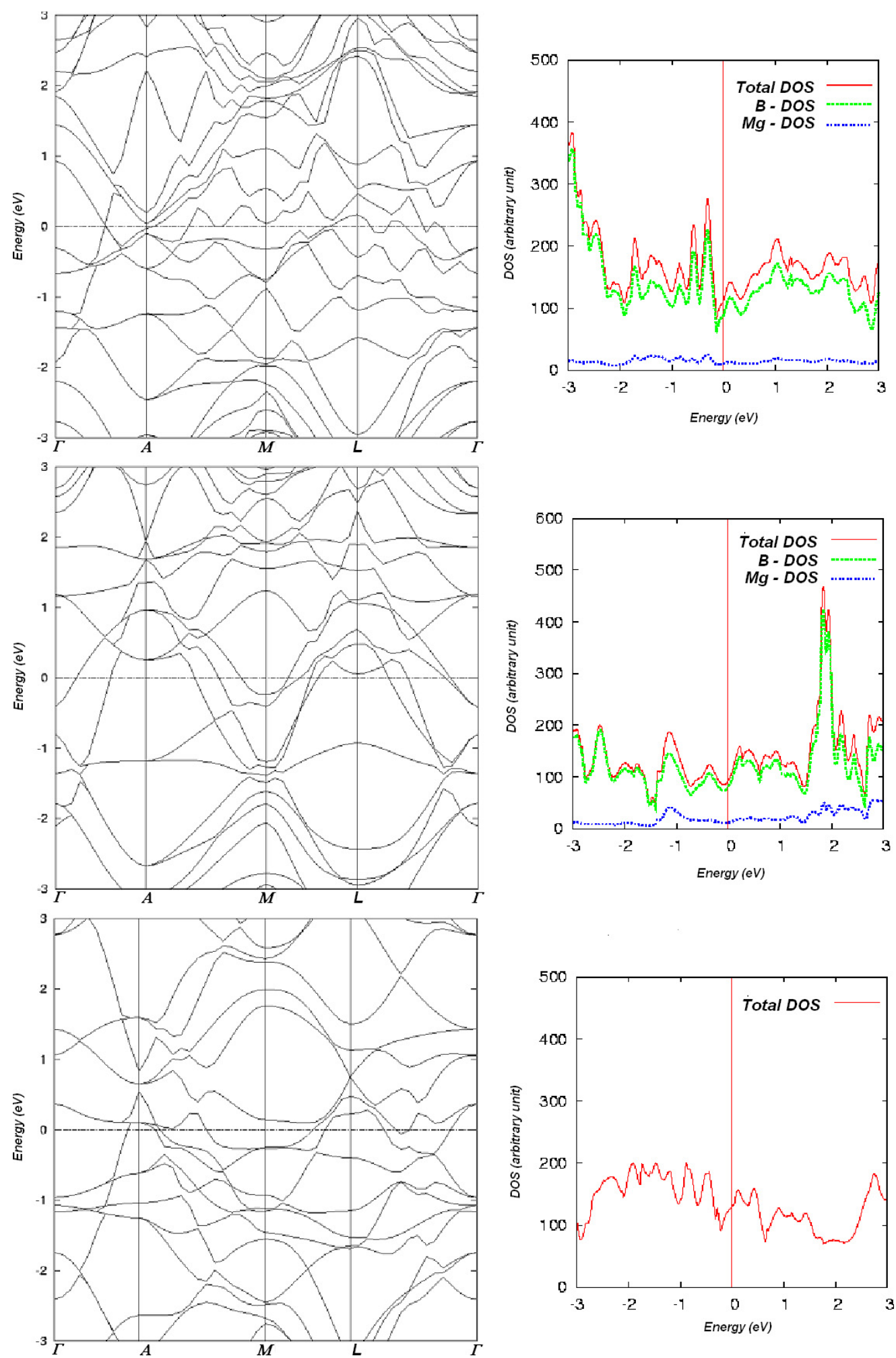

Figure 5. Band structure and density of states: top, $\mathrm{Mg}_{5} \mathrm{~B}_{24}$; center, $\mathrm{MgB}_{8}$; bottom, pristine SWBNT bundles. The Fermi level is shifted to $0 \mathrm{eV}$.

The band structure of the Mg-intercalated bundles along several unique $k$-directions (i.e. $\Gamma-\mathrm{A}-\mathrm{M}-\mathrm{L}-\Gamma$ ) is given in figure 5. It shows highly anisotropic features along the tubular axis $(\Gamma-\mathrm{A})$ and the intertubular $(\mathrm{A}-\mathrm{M}-\mathrm{L}-\Gamma)$ direction which are similar to those of the pristine SWBNT bundles. The (partially occupied) conduction bands crossing $E_{\mathrm{F}}$ are attributed to $\mathrm{s}-\mathrm{p}$ hybridized and $\mathrm{p}_{x, y}$ characters associated with the $\pi$-like bonds between the intertubules. Their presence explains a stronger coupling of the tubule networks in the bundles yielding boron $\mathrm{p}$ features in density of states around $E_{\mathrm{F}}$. On the other hand, the occupied valence bands are formed by the intertubular boron p-type $\sigma$ bonds, and intratubular 


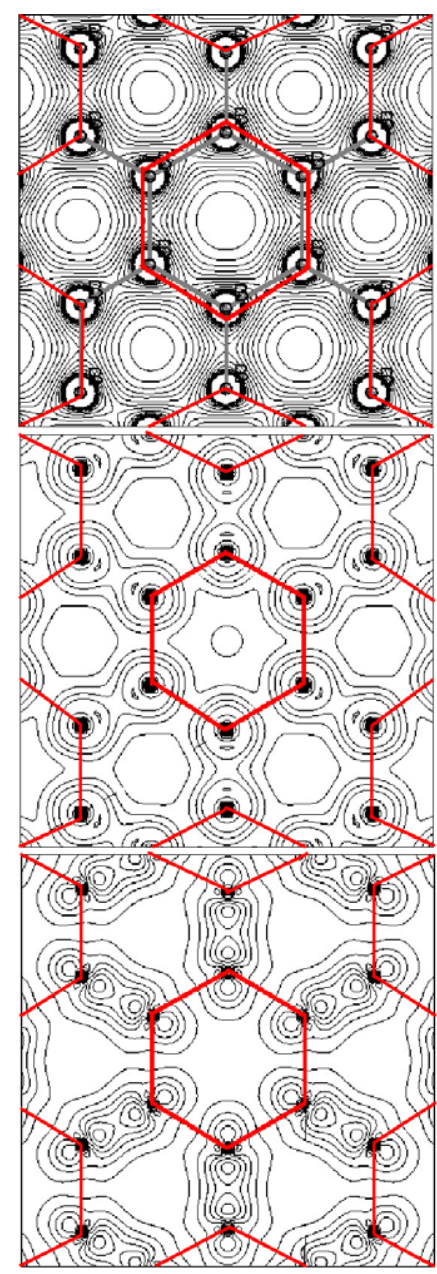

SWBNTs Bundle

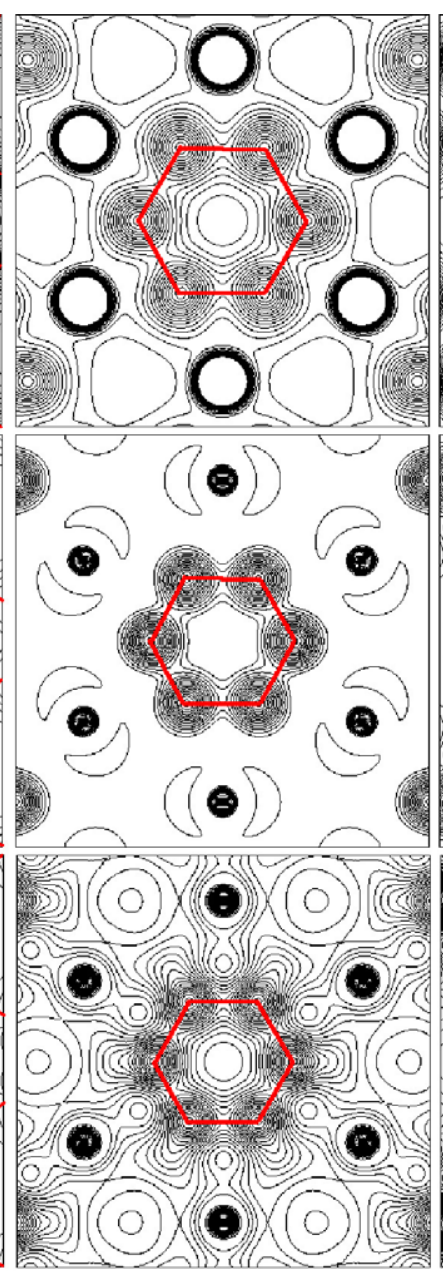

$\mathrm{MgB}_{8}$

SWBNTs Bundle

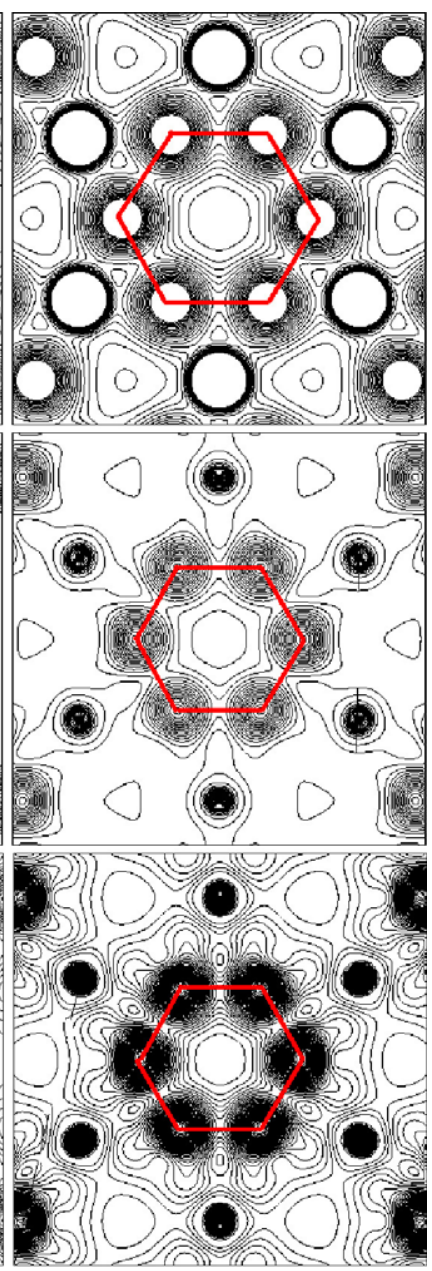

$\mathrm{Mg}_{5} \mathrm{~B}_{24}$

SWBNTs Bundle

Figure 6. A 2D contour map of the electron charge density plot for 3D crystalline nanotube bundles. The red lines (gray lines in the printed version) correspond to the outlines of the hexagonal shape of the SWBNT structure along the tubular axis in the top view. Total charge density (top row), occupied valence (center row) and partially occupied conduction (bottom row) states near the Fermi energy of SWBNT bundles and Mg-intercalated bundles, $\mathrm{MgB}_{8}$ and $\mathrm{Mg}_{5} \mathrm{~B}_{24}$.

Table 1. Binding energy of $\mathrm{Mg}$ at the $\mathrm{H}$ site on the surface of a pristine SWBNT. $\Theta$ is the coverage of $\mathrm{Mg}$ on the tubular surface, $E_{\mathrm{b}}$ is the binding energy, $E_{\mathrm{s}}$ is the binding energy per $\mathrm{Mg}$ atom and $R_{\mathrm{Mg}-\mathrm{B}}$ is the distance between $\mathrm{Mg}$ and $\mathrm{B}$ in the tubular configuration, compared to the $\mathrm{Mg}-\mathrm{B}$ bond distance (i.e. $2.51 \AA$ ) in $\mathrm{MgB}_{2}$ solid [19].

\begin{tabular}{lllll}
\hline $\begin{array}{l}\text { Number of } \\
\text { Mg atoms } \\
\text { (per unit cell) }\end{array}$ & $\begin{array}{l}\Theta(\mathrm{Mg}: \mathrm{B}) \\
\text { (per unit }\end{array}$ & $\begin{array}{l}R_{(\mathrm{Mg}-\mathrm{B})} \\
(\AA)\end{array}$ & $\begin{array}{l}E_{\mathrm{b}} \\
(\mathrm{eV})\end{array}$ & $\begin{array}{l}E_{\mathrm{s}} \\
(\mathrm{eV} / \mathrm{Mg} \text { atom })\end{array}$ \\
\hline $1 \mathrm{Mg}$ & $1: 24$ & 2.38 & 1.55 & 1.55 \\
$2 \mathrm{Mg}$ & $1: 12$ & 2.38 & 3.01 & 1.51 \\
$3 \mathrm{Mg}$ & $1: 8$ & 2.39 & 4.53 & 1.51 \\
$4 \mathrm{Mg}$ & $1: 6$ & $2.34,2.38^{\mathrm{a}}$ & 5.48 & 1.37 \\
$6 \mathrm{Mg}$ & $1: 4$ & 2.56 & 6.49 & $1.08^{\mathrm{a}}$ \\
$12 \mathrm{Mg}$ & $1: 2$ & 2.53 & 17.87 & 1.49 \\
\hline
\end{tabular}

${ }^{a}$ The minor differences in distance between $\mathrm{Mg}$ and $\mathrm{B}$ (i.e. $R_{\mathrm{Mg}-\mathrm{B}}$ ) are due to the asymmetric $\mathrm{Mg}$ atom coverage (i.e. $\Theta=\frac{1}{6}$ ) at the $\mathrm{H}$ binding site on the tubular surface. For $\Theta=\frac{1}{4}$, the low $E_{\mathrm{s}}$ is due to larger $R_{\mathrm{Mg}-\mathrm{B}}$ relative to the other $\Theta$ values considered. boron p-type $\sigma$ and $\pi$ bonds. As $\mathrm{Mg}$ atoms settle down at the interstitial sites in the bundles, the nature of the density of states near $E_{\mathrm{F}}$ remains the same, predominantly associated with boron

Table 2 shows that $E_{\mathrm{s}}$ remains nearly constant, approaching $75 \%$ of the intercalation energy of about $4.1 \mathrm{eV}$ for crystalline $\mathrm{MgB}_{2}$. Interestingly, $E_{\mathrm{s}}$ of the $\mathrm{Mg}-\mathrm{SWBNT}$ bundle is almost twice the value of $E_{\mathrm{s}}$ of pristine SWBNTs (table 1). Since Mulliken charge analysis does not indicate a larger charge transfer from $\mathrm{Mg}$ in $\mathrm{Mg}-\mathrm{SWBNT}$ bundles relative to that in pristine SWBNTs, the additional stability of $\mathrm{Mg}$-intercalated bundles appears to rise from the topological distribution of charge over the local (i.e. tubules) and crystalline (i.e. intertubular and interstitial) region. In fact, one can say that the $\mathrm{Mg}$ atoms are partially ionized in the crystallite bundles and the electrons donated to the system are not localized, but rather are effectively distributed over the lattice as shown by the 2D contour map of total and partial charge density near $E_{\mathrm{F}}$ in figure 6. Consequently, 
Table 2. Binding energy of the intercalated $\mathrm{Mg}$ in (small diameter) SWBNT crystalline bundles. $\Theta$ is the intercalation density, $E_{\mathrm{b}}$ is the binding energy, $E_{\mathrm{s}}$ is the intercalation energy $\left(E_{\mathrm{b}} / \mathrm{Mg}\right.$ atoms in the unit cell), $R_{\mathrm{Mg}-\mathrm{B}}$ is the distance between $\mathrm{Mg}$ and $\mathrm{B}$.

\begin{tabular}{llllllll}
\hline System & $\begin{array}{l}\Theta(\mathrm{Mg}: \mathrm{B}) \\
\text { (unit cell) }\end{array}$ & $\begin{array}{l}\text { Volume } \\
(\AA)^{3}\end{array}$ & $\begin{array}{l}\text { Lattice parameters } \\
a, c(\AA)\end{array}$ & $\begin{array}{l}R_{\mathrm{Mg}-\mathrm{B}} \\
(\AA)\end{array}$ & $\begin{array}{l}R_{\text {intertube }} \\
(\AA)\end{array}$ & $\begin{array}{l}E_{\mathrm{b}} \\
(\mathrm{eV})\end{array}$ & $\begin{array}{l}E_{\mathrm{s}} \\
(\mathrm{eV} / \mathrm{Mg})\end{array}$ \\
\hline Pristine SWBNT bundle & - & 197.91 & $6.21,5.93$ & - & 1.94 & - & - \\
Mg-SWBNT bundle & $1: 8$ & 309.51 & $7.79,5.89$ & 2.52 & 3.73 & 9.03 & 3.01 \\
Mg-SWBNT bundle & $5: 24$ & 298.10 & $7.83,5.61$ & 2.39 & 3.41 & 14.30 & 2.86 \\
\hline
\end{tabular}

instead of the dominance of the intertubular $(2 c)$ covalent bonds in the bundles (figure 6), the lattice can be imagined as an 'electrostatic' bound system consisting of the cation (i.e. partially ionized $\mathrm{Mg}$ ) and anion (i.e. partially ionized tubules) in the Mg-SWBNT bundles considered. The metallic character of the tubular $\mathrm{Mg}-\mathrm{B}$ bundles, therefore, can be attributed to boron atoms forming a metallic wire, while the role of $\mathrm{Mg}$ atoms is limited to enhancing the stability of the crystalline bundles.

\section{Summary}

Mg-intercalated single-walled boron nanotubes together with their crystalline bundles can be considered as a novel boronbased compounds which are closely related to $\mathrm{MgB}_{2}$ metaldiboride crystalline solids [19, 20] and nanostructures [22-24]. The calculated results reveal a highly selective adsorption site for $\mathrm{Mg}$ atoms on the tubule walls. Analysis of the chemical bonding reveals that $\mathrm{Mg}$ is partially ionized, and the charge transfer from $\mathrm{Mg}$ to the $\mathrm{B}$ sublattice is not localized on the boron atoms, but rather is distributed over the tubular surface of Mg-SWBNTs or the interstitial regions of the Mg-SWBNT bundles.

The calculated results predict the intrinsic relationship between $\Theta=\frac{1}{2}$ coverage of $\mathrm{Mg}$ on the surface of a SWBNT and single layer $\mathrm{MgB}_{2}$. The curvature energy of a $(6,0)$ zigzag $\mathrm{MgB}_{2}$ single-walled nanotube is found to be about $0.13 \mathrm{eV} /$ atom relative to the equilibrium configuration of a $\mathrm{MgB}_{2}$ sheet. The calculated results, therefore, suggest relatively easier synthesis of $\mathrm{MgB}_{2}$ singlewalled nanotubes [22] under appropriate conditions.

\section{Acknowledgments}

This work was partially supported by DARPA through ARL contract no. DAAD17-03-C-0115. We thank S Gowtham and Haiying He for helpful comments during this work.

\section{References}

[1] Boustani I and Quandt A 1997 Europhys. Lett. 39527

[2] Gindulytu A, Lipscomb W N and Massa L 1998 Inorg. Chem. 376544

[3] Boustani I, Quandt A, Hernández E and Rubio A 1999 J. Chem. Phys. 1103176

[4] Evans M H, Joannopoulos J D and Pantelides S T 2005 Phys. Rev. B 72045434

[5] Lau K C, Pati R, Pandey R and Pineda A C 2006 Chem. Phys. Lett. 418549

[6] Lau K C, Pandey R, Pati R and Karna S P 2006 Appl. Phys. Lett. 88212111

[7] Kunstmann J and Quandt A 2006 Phys. Rev. B 74035413

[8] Cabria I, López M J and Alonso J A 2006 Nanotechnology 17778

[9] Lau K C and Pandey R 2007 J. Phys. Chem. C 1112906

[10] Tang H and Ismail-Beigi S 2007 Phys. Rev. Lett. 99115501

[11] Kunstmann J and Quandt A 2005 Chem. Phys. Lett. 40221

[12] Lau K C, Orlando R and Pandey R 2008 J. Phys.: Condens. Matter 20125202

[13] Zhao J, Buldum A, Han J and Lu J P 2000 Phys. Rev. B 851706

[14] Ciuparu D, Klie R F, Zhu Y and Pfefferle L 2004 J. Phys. Chem. B 1083967

[15] Perdew J P and Wang Y 1992 Phys. Rev. B 4513244

[16] Saunders V R, Dovesi R, Roetti C, Orlando R, Zicovich-Wilson C M, Harrison N M, Doll K, Civalleri B, Bush I J, D' Arco P and Llunell M 2006 CRYSTAL06 User's Manual Università di Torino, Torino

[17] Jona F and Marcus P M 2002 Phys. Rev. B 66094104

[18] Lau K C and Pandey R 2008 J. Phys. Chem. B 11210217

[19] Prassides K, Iwasa Y, Ito T, Chi D H, Uehara K, Nishibori E, Takata M, Sakata M, Ohishi Y, Shimomura O, Muranaka T and Akimitsu J 2001 Phys. Rev. B 64012509

[20] Nagamatsu J, Nakagawa N, Muranaka T, Zenitani Y and Akimitsu J 2001 Nature 41063

[21] Bagci V M, Gulseren O, Yildirim T, Gedik Z and Ciraci S 2002 Phys. Rev. B 66045409

[22] Chernozatonskii L A 2001 JETP Lett. 74335

[23] Bamburov V G, Ivanovskaya V V, Enyashin A N, Shein I R, Medvedeva N I, Makurin Y N and Ivanovskii A L 2003 Dokl. Phys. Chem. 38843

[24] Nath M and Parkinson B A 2006 Adv. Mater. 181865 